\title{
Estudo do desempenho de reator de leito gotejante aplicado ao processo de hidrodessulfurização do petróleo
}

Study of the performance of a trickle bed reactor applied to oil hydrodesulfurization process

\author{
M. F. F. Souza ${ }^{1 *}$; P. L. Santana ${ }^{2}$ \\ ${ }^{1}$ Departamentode Química/Campus Itabaiana, Universidade Federal de Sergipe, 49500-000, Itabaina-SE, Brasil \\ ${ }^{2}$ Departamento de Engenharia Química/Centro Ciências Exatas, Universidade Federal de Sergipe, 49100-000, \\ Aracaju-SE,Brasil
}

*marcos_ffsouza@hotmail.com

(Recebido em 13 de março de 2016; aceito em 15 de abril de 2016)

\begin{abstract}
Um modelo de reator heterogêneo unidimensional em regime transiente foi desenvolvido para simular um reator de leito gotejante aplicado à hidrodessulfurização de petróleo. O modelo incorpora a resistência à transferência de massa nas interfaces gás-líquido e líquido-sólido e uma expressão da taxa da reação baseada no modelo cinético do tipo Langmuir-Hinshelwood. Mediante estimativa de parâmetros, o modelo foi ajustado com base em dados experimentais de um reator em escala piloto e, então, validado sob diferentes condições de operação. Por fim, estudou-se tanto o efeito de inibição de $\mathrm{H}_{2} \mathrm{~S}$ como também o efeito das condições operacionais sobre a conversão de HDS, tais como: pressão, temperatura, velocidade espacial horária líquida (LHSV).
\end{abstract}

Palavras-chave: reator de leito gotejante, hidrodessulfurização, modelo heterogêneo

A heterogeneous reactor model in one-dimensional transient was developed to simulate a trickle bed reactor (TBR) applied to hydrodesulfurization (HDS) of oil. The model incorporates the resistance to mass transfer in gas-liquid and liquid-solid interfaces and an expression of the reaction rate based kinetic model of Langmuir-Hinshelwood type. Through parameter estimation, the model was fitted based on experimental data from a pilot-scale reactor and then validated under different experimental conditions. Finally, it were studied both $\mathrm{H}_{2} \mathrm{~S}$ inhibiting effect and the effect of operating conditions on the HDS conversion, such as pressure, temperature, and liquid hourly space velocity (LHSV).

Keywords: trickle bed reactor, hydrodesulfurization, heterogeneous model

\section{INTRODUÇÃo}

A demanda por energia no mundo é crescente e atingiu um aumento de 0,9\% em 2014 devido ao rápido crescimento dos países emergentes, tais como China e Índia. Enquanto isso, o petróleo permanece em destaque como a principal fonte de combustível no mundo, representando 32,6\% do consumo de energia global [1].

Diante disso, a busca por novas jazidas de petróleo tem resultado em crescimento no volume mundial de petróleos pesados, com menor rendimento em derivados leves. Essa evolução das reservas tem indicado uma tendência à oferta de petróleo mais pesado e com maior teor de enxofre [2].

Esses petróleos são atualmente processados nas refinarias para obtenção de derivados combustíveis, em que a combustão desses contribui amplamente para à geração de poluentes atmosféricos, tais como os óxidos de enxofre (SOx) [3, 4]. Além do mais, a presença desses contaminantes torna prejudicial à eficiência e segurança do processo, já que podem provocar tanto a corrosão de equipamentos quanto o envenenamento de catalisadores na refinaria [5].

Nesse sentido, a remoção de compostos heteroátomos do petróleo é necessária para adequá-lo às condições de processamento na refinaria, além de satisfazer às regulamentações ambientais cada vez mais rigorosas de seus derivados combustíveis. Para tanto, a remoção desses compostos do petróleo é realizada mediante o uso de um dos processos mais importantes na refinaria de petróleo, o processo de hidrotratamento [6, 7]. Nesse processo, frações de petróleo são 
submetidas a altas temperaturas e pressões de hidrogênio para a redução do teor de contaminantes [8].

Várias tecnologias, tais como reatores catalíticos trifásicos, estão disponíveis para o hidroprocessamento de frações de petróleo. Entre essas tecnologias, aquela usando reatores de leito gotejante ou TBR (do inglês trickle bed reactor) se tornou bastante utilizada na indústria petrolífera, mais especificamente para a promoção da hidrodessulfurização ou HDS (do inglês hydrodesulfurization). Os reatores de leito gotejante se caracterizam pelo fluxo das correntes de gás e líquido ocorrerem de modo descendente através de um leito catalítico [9].

$\mathrm{Na}$ literatura, encontram-se revisões que abordam a hidrodessulfurização (HDS) de compostos modelos [10], frações de petróleo [11] e petróleo pesado [12] com interesse nas características mais importantes daquele processo, que são a cinética e a modelagem do reator. Diferentes abordagens cinéticas têm sido utilizadas sendo a mais comum o modelo cinético agrupado, que tentar modelar a HDS de petróleo e estabelecer a dependência dessa reação com as variáveis de processo $[13,14,15]$.

Entre as abordagens de modelagem de reatores químicos envolvendo reações de hidrotratamento, as mais comuns são aquelas que fazem a análise baseada no modelo a parâmetros concentrados, geralmente desenvolvidos como modelos de reatores pseudohomogêneo e heterogêneo em estado estacionário, enquanto que estudos sobre modelagem e simulação transiente sejam com modelos de reatores pseudo-homogêneos ou heterogêneos são escassos [16].

O presente trabalho visa estudar o processo de hidrodessulfurização em reatores de leito gotejante, como técnica para o tratamento de petróleo. Tal estudo consistirá na proposição de um modelo matemático heterogêneo unidimensional em regime transiente, no qual são considerados os balanços de conservação (massa e energia), fenômenos de transferência de massa entre fases e cinética química heterogênea. Para a validação do modelo foram utilizados dados experimentais disponíveis na literatura para um processo de hidrodessulfurização em escala piloto [17].

\section{MATERIAL E MÉTODOS}

Inicialmente, realizou-se o cálculo dos parâmetros associados com as equações de balanço de massa e energia no reator. Para isso, foram utilizados os dados experimentais referentes às propriedades da alimentação de petróleo e gás hidrogênio e as dimensões do reator disponíveis no estudo de [17], que são listados na Tabela 1. Nesse estudo, os experimentos foram conduzidos em um reator de leito gotejante em escala piloto usando petróleo bruto como alimentação e um catalisador comercial típico para $\mathrm{HDS}\left(\mathrm{Co}-\mathrm{Mo} / \mathrm{Al}_{2} \mathrm{O}_{3}\right)$, cujas propriedades são apresentadas na Tabela 2. Essas propriedades são necessárias a fim de calcular o fator de efetividade do catalisador. A Tabela 3 lista as conversões experimentais em diferentes condições operação obtida por [17], que foram utilizadas para validar o modelo do reator estado transiente proposto neste trabalho.

Tabela 1: Propriedades da alimentação e as dimensões do reator TBR em escala piloto [17].

\begin{tabular}{lc}
\hline \multicolumn{1}{c}{ Parâmetro } & Valor \\
\hline Massa molecular do petróleo $(\mathrm{g} / \mathrm{mol})$ & 227,5 \\
Diâmetro interno no reator $(\mathrm{m})$ & 0,004 \\
Comprimento do reator $(\mathrm{m})$ & 0,65 \\
Comprimento do leito catalítico $(\mathrm{m})$ & 0,278 \\
Teor de enxofre (\%) & 2,0 \\
Gravidade específica do petróleo a $15,6^{\circ} \mathrm{C}($ (adimensional) & 0,8558 \\
Ponto de ebulição médio da alimentação $\left({ }^{\circ} \mathrm{C}\right)$ & 291 \\
Razão $\mathrm{H}_{2} /$ óleo (L/L) & 250 \\
\hline
\end{tabular}


Tabela 2: Propriedades do catalisador [17].

\begin{tabular}{lc}
\hline \multicolumn{1}{c}{ Parâmetro } & Valor \\
\hline Densidade do leito catalítico $\left(\mathrm{kg} / \mathrm{m}^{3}\right)$ & 670 \\
Diâmetro equivalente da partícula $(\mathrm{mm})$ & 4 \\
Área específica $\left(\mathrm{m}^{2} / \mathrm{g}\right)$ & 180 \\
Volume de poro $\left(\mathrm{cm}^{3} / \mathrm{g}\right)$ & 0,5 \\
Tortuosidade & 4 \\
\hline
\end{tabular}

Tabela 3: Conversão de HDS experimental sob diferentes condições de operação [17].

\begin{tabular}{cccc}
\hline $\mathbf{T}$ & $\mathbf{P}$ & $\mathbf{L H S V}$ & Conversão Experimental HDS \\
$(\mathbf{(} \mathbf{C})$ & $(\mathbf{M P a})$ & $\left(\mathbf{h}^{\mathbf{- 1}}\right)$ & 78,50 \\
\hline 335 & 10 & 0,5 & 90,20 \\
370 & 10 & 0,5 & 95,90 \\
400 & 10 & 0,5 & 93,25 \\
400 & 7 & 0,5 & 88,50 \\
400 & 4 & 0,5 & 81,20 \\
400 & 10 & 1,0 & 68,00 \\
400 & 10 & 1,5 & \\
\hline
\end{tabular}

\section{Reações Químicas e Modelo Cinético}

Comumente, o teor de enxofre em qualquer alimentação varia entre $0,1 \%$ a $8 \%$ em massa [18]. O enxofre pode ser encontrado na forma de mercaptanas, sulfetos, dissulfetos e tiofenos, etc [19].

Essa diferença estrutural entre as várias moléculas contendo enxofre presentes em frações de petróleo torna impraticável ter uma expressão de taxa simples. Visto que cada molécula de enxofre tem sua própria cinética de hidrogenólise e na maioria das vezes não é simples extrair efeitos individuais de cada composto [20].

A expressão cinética de HDS mais frequentemente utilizada é o modelo de LangmuirHinshelwood:

$$
r_{H D S}^{\prime}=\frac{k_{H D S} C_{e n x}^{S n} C_{H_{2}}^{S m}}{\left(1+K_{H_{2} S} C_{H_{2} S}^{S}\right)^{2}}
$$

As ordens de reação para enxofre e hidrogênio, $n$ e $m$, respectivamente, são ajustáveis de acordo com a alimentação e podem variar entre 1,5 e 2,5 para o enxofre e entre 0,5 e 1,0 para o hidrogênio [20].

A constante de equilíbrio de adsorção e a constante taxa cinética são baseadas na equação de Arrhenius:

$K_{H_{2} S}=K_{0} \exp \left(\frac{\Delta H_{a d s}}{R T}\right)$

onde $K_{0}$ é o fator pré-exponencial em $\mathrm{m}^{3} \mathrm{~mol}^{-1}, \Delta H_{a d s}$ é a entalpia de adsorção em $\mathrm{J} \mathrm{mol}^{-1}$.

\section{Modelo do Reator}

Primeiramente, um modelo de reator de leito gotejante baseado na abordagem unidimensional heterogênea em estado transiente foi desenvolvido neste trabalho. As principais hipóteses para a simplificação da modelagem foram as seguintes: (1) regime transiente; (2) as fases gás e líquida apresentam fluxo empistonado; (3) operação adiabática; (4) evaporação e condensação desprezível da fase líquida; (5) reação química apenas na fase líquida em contato com a fase sólida; (6) holdups das fases líquido e gás no reator são constantes; (7) atividade do catalisador não se altera com o tempo; (8) fase sólida isotérmica; (9) balanço na fase gás é desprezível, já que a capacidade calorífica dessa fase é muito menor comparada aquela da fase líquida.

As equações de balanço de massa para cada componente nas fases gás, líquida e sólida são apresentadas a seguir: 
Fase gás $\left(i=H_{2}\right.$ e $\left.H_{2} S\right)$ :

$\frac{\varepsilon_{G}}{R T} \frac{\partial p_{i}^{G}}{\partial t}=-\frac{u_{G}}{R T} \frac{\partial p_{i}^{G}}{\partial z}-k_{i}^{L} a_{L}\left(\frac{p_{i}^{G}}{H_{i}}-C_{i}^{L}\right)$

Fase líquida $\left(i=\mathrm{H}_{2}\right.$ e $\left.\mathrm{H}_{2} \mathrm{~S}\right)$ :

$\varepsilon_{L} \frac{\partial C_{i}^{L}}{\partial t}=-u_{L} \frac{\partial C_{i}^{L}}{\partial z}+k_{i}^{L} a_{L}\left(\frac{p_{i}^{G}}{H_{i}}-C_{i}^{L}\right)-k_{i}^{S} a_{S}\left(C_{i}^{L}-\left.C_{i}^{S}\right|_{r=R}\right)$

Fase líquida $(i=e n x)$ :

$\varepsilon_{L} \frac{\partial C_{e n x}^{L}}{\partial t}=-u_{L} \frac{\partial C_{e n x}^{L}}{\partial z}-k_{i}^{S} a_{S}\left(C_{e n x}^{L}-C_{e n x}^{S}\right)$

As equações de balanço de massa para o hidrocarboneto não foram consideradas, pois a concentração desse não muda significativamente durante a reação de HDS [21].

Fase sólida $\left(i=H_{2}, H_{2} S\right.$ e enx $)$ :

$\varepsilon_{P}\left(1-\varepsilon_{B}\right) \frac{d C_{i}^{S}}{d t}=k_{i}^{S} a_{S}\left(C_{i}^{L}-C_{i}^{S}\right)+v_{i} \rho_{B} \eta_{H D S} r_{H D S}^{\prime}$

Uma vez que as reações de HDS são de natureza exotérmica, as equações de balanço de energia para o reator, operando em condição não isotérmica e transiente, são apresentadas a seguir:

Fase líquida:

$\varepsilon_{L} \rho_{L} C_{P_{L}} \frac{\partial T_{L}}{\partial t}=-u_{L} \rho_{L} C_{P_{L}} \frac{\partial T_{L}}{\partial z}-h_{L S} a_{S}\left(T_{L}-T_{S}\right)$

Fase sólida:

$\varepsilon_{S} \rho_{S} C_{P_{S}} \frac{d T_{S}^{S}}{d t}=h_{L S} a_{S}\left(T_{L}-T_{S}^{S}\right)+\rho_{B}\left(-\Delta H_{R, H D S}\right) \eta_{H D S} r_{H D S}^{\prime}$

Os balanços de massa e energia apresentados correspondem a um sistema de equações diferenciais parciais (EDP), que foi resolvido usando o método das linhas. Esse método consiste em transformar as EDPs em um conjunto de equações diferenciais ordinárias (EDO) de primeira ordem pela discretização das derivadas espaciais na direção axial usando expressão de diferença finita atrasada e mantendo as derivadas parciais no tempo sem discretizar [16].

Nesse sentido, um código computacional foi desenvolvido para solução numérica do modelo TBR no software Matlab com base em códigos apresentados por [22], que estabeleceram procedimentos numéricos envolvendo o método das linhas para resolução de EDPs em linguagem Matlab. As principais características do modelo foram: 100 nós de discretização, que foram requeridos para obter uma precisão razoável; o tempo de integração foi longo o suficiente para atingir as condições de estado estacionário; a função ode15i do Matlab para resolução de EDOs rígidas foi utilizada; e a aplicação da expressão de diferença finita atrasada às derivadas parciais espaciais na direção axial. 


\section{RESULTADOS E DISCUSSÃO}

Uma vez que todas as equações para o balanço de massa e energia foram definidas e todos os parâmetros foram calculados, o modelo do reator pode ser validado. Para isso, realizou-se uma comparação entre os resultados de conversão de enxofre preditos pelo modelo e os dados de conversão experimental disponível em [17]. A validação resultou em um erro relativo variando entre $1,15 \%$ e $9,43 \%$. Diante desse resultado, pode-se perceber que o modelo matemático conseguiu reproduzir quantitativamente bem os resultados de conversão de HDS experimentais.

Uma vez que o modelo foi validado, prosseguiu-se o estudo dos perfis de pressão parcial, concentração e temperatura sob condições de processo selecionadas e os efeitos das variáveis operacionais sobre a conversão na HDS.

\section{Perfis de Pressão Parcial e Concentração de $\mathbf{H}_{2}$}

Na Figura 1, apresenta-se os perfis de pressão e concentração de hidrogênio ao longo do eixo do reator. Todos os gráficos foram gerados em $400^{\circ} \mathrm{C}, 10 \mathrm{MPa}$, LHSV $=1,39 \mathrm{E}-4 \mathrm{~s}^{-1}\left(0,5 \mathrm{~h}^{-1}\right)$ e $\varphi$ $=250 \mathrm{~m}^{3} / \mathrm{m}^{3}$ de alimentação.
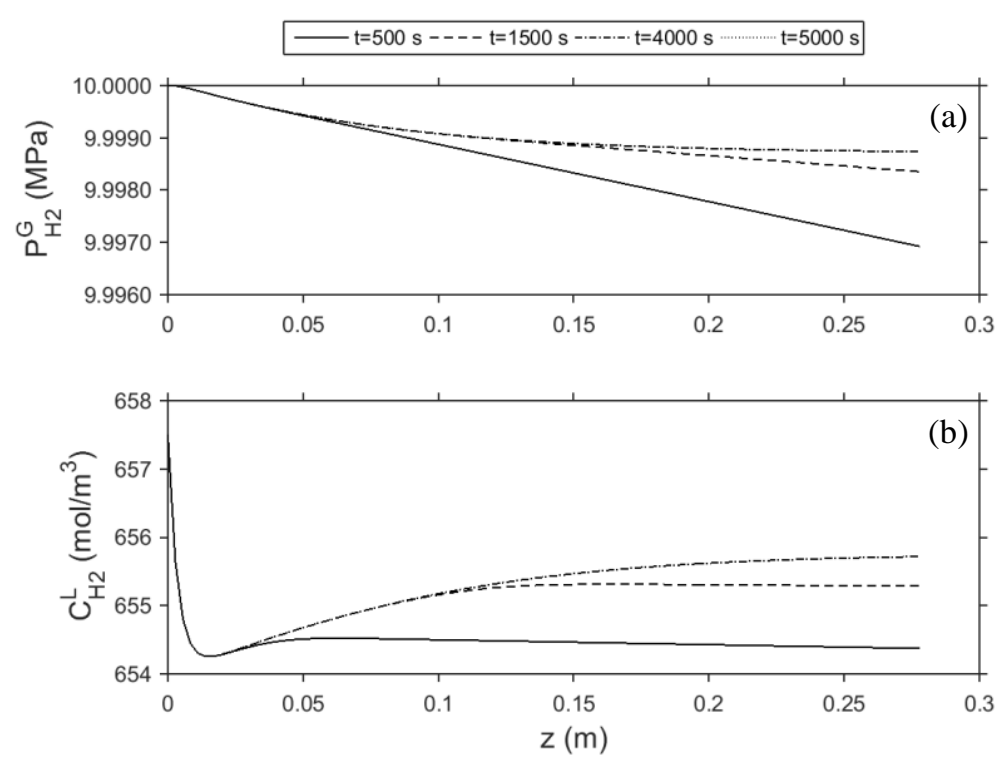

Figura 1: Pressão parcial (a) e concentração molar de hidrogênio (b) ao longo do comprimento do leito catalítico no reator em diferentes tempos.

Como observado, a pressão parcial de hidrogênio decai à medida que percorre o comprimento do leito catalítico ao longo dos diferentes tempos analisados. A concentração de hidrogênio na fase líquida mostra um decaimento da na seção inicial do reator, ou seja, apresenta gradiente de concentração negativo. Esse efeito é decorrente do termo relacionado à taxa de transferência de massa ser menor do que o termo relacionado à taxa de reação química, tal que a concentração de $\mathrm{H}_{2}$ na fase líquida é esgotada e quase todo $\mathrm{H}_{2}$ está na fase gás. Por outro lado, conforme a reação avança, a taxa de transferência de massa é predominante sobre o termo da taxa de reação química e uma elevada quantidade de $\mathrm{H}_{2}$ é transferida da fase gás para fase líquida, aumentando a concentração na fase líquida.

\section{Perfis de Pressão Parcial e Concentração de $\mathrm{H}_{2} \mathrm{~S}$}

Na Figura 2, mostra-se o perfil transiente de sulfeto de hidrogênio na fase gás e líquida ao longo do comprimento do leito. 

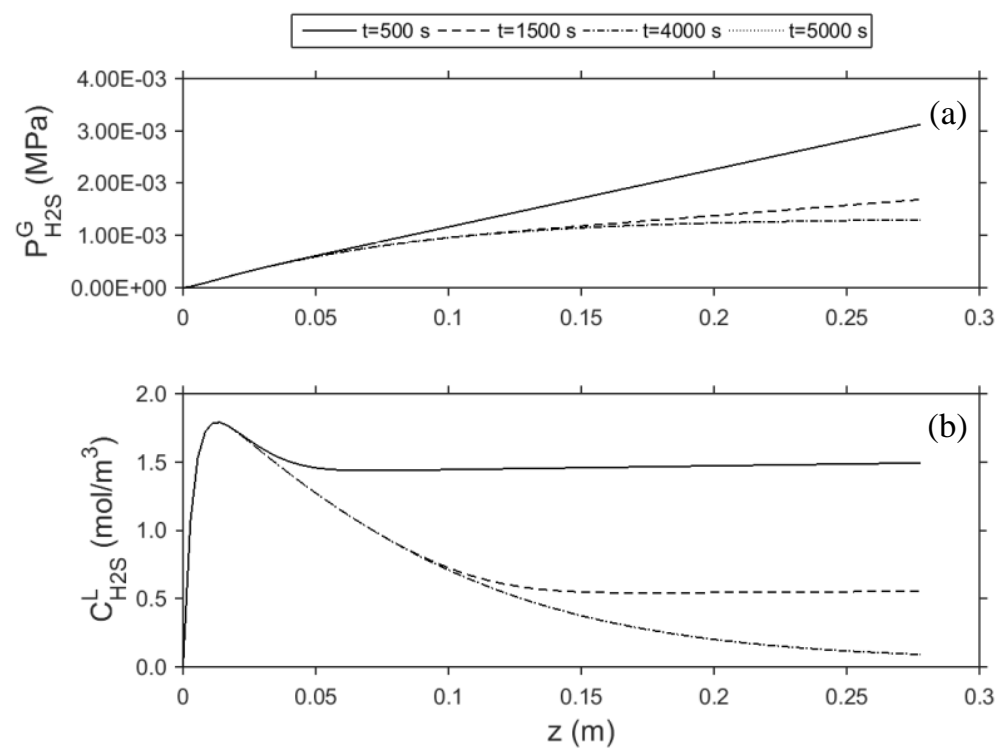

Figura 2: Pressão parcial (a) e concentração molar de $\mathrm{H}_{2} \mathrm{~S}(b)$ ao longo do leito catalítico no reator em diferentes intervalos de tempo.

Na entrada do reator não há $\mathrm{H}_{2} \mathrm{~S}$, mas ao longo do leito catalítico se nota elevação da pressão parcial de $\mathrm{H}_{2} \mathrm{~S}$ decorrente da sua geração durante a reação. Por outro lado, com o passar do tempo ocorre uma redução do gradiente de concentração de $\mathrm{H}_{2} \mathrm{~S}$ após a seção inicial do reator, devido ao efeito de inibição do $\mathrm{H}_{2} \mathrm{~S}$ na taxa de reação, até atingir a condição estacionária. $\mathrm{O}$ perfil de concentração de $\mathrm{H}_{2} \mathrm{~S}$ na fase líquida apresenta comportamento análogo e oposto àquele de observado para o $\mathrm{H}_{2}$ (Figura 2b), já que o $\mathrm{H}_{2} \mathrm{~S}$ é liberado da fase líquida para fase gás durante a HDS.

\section{Perfis de Concentração de Enxofre na Fase Líquida}

Na Figura 3 são apresentados os perfis das concentrações de enxofre na fase líquida ao longo do comprimento do leito catalítico em diferentes intervalos de tempo. A concentração de enxofre mostra um decaimento com a evolução do tempo.

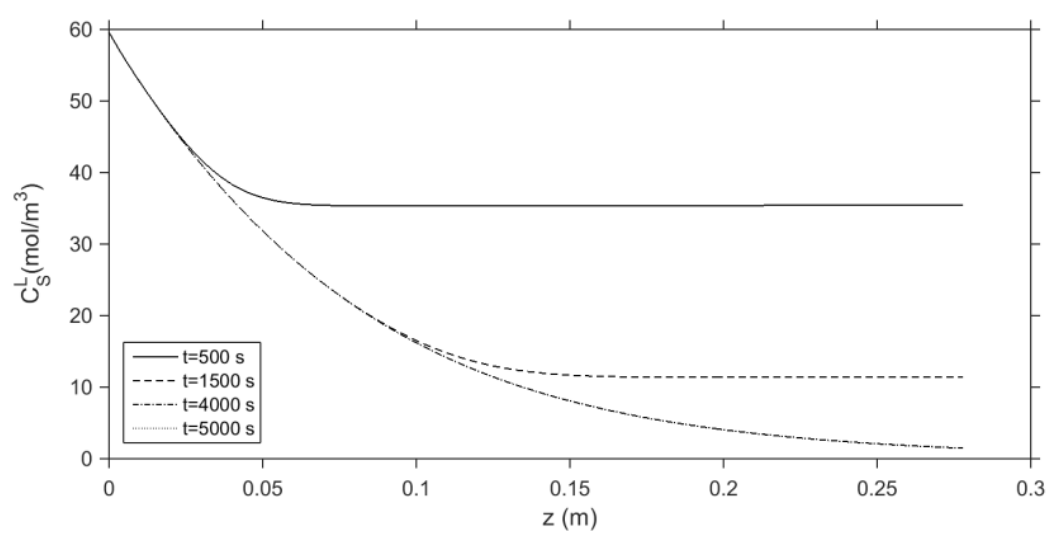

Figura 3: Concentração de compostos organossulfurados ao longo do comprimento do leito catalítico no reator em diferentes intervalos de tempo.

\section{Perfis de Temperatura nas fases líquida e sólida}

A Figura 4 mostra a evolução de temperatura na fase líquida e sólida ao longo do reator em diferentes tempos de operação. No tempo mais curto (500 s), a temperatura aumenta ligeiramente no início do leito e logo após se mantém constante. À medida que o tempo aumenta $(1500 \mathrm{~s} \mathrm{e}$ 
3000 s) há um progressivo aumento da temperatura até se atingir a condição de regime estacionário no tempo de $5000 \mathrm{~s}$. Nessa condição, a elevação de temperatura foi de quase $7 \mathrm{~K}$, alcançando 679,65 K na saída do leito catalítico. Nota-se também que os perfis de temperatura das fases líquida e sólida são semelhantes, o que permitiria a simulação do balanço de energia apenas com uma equação de pseudofase.
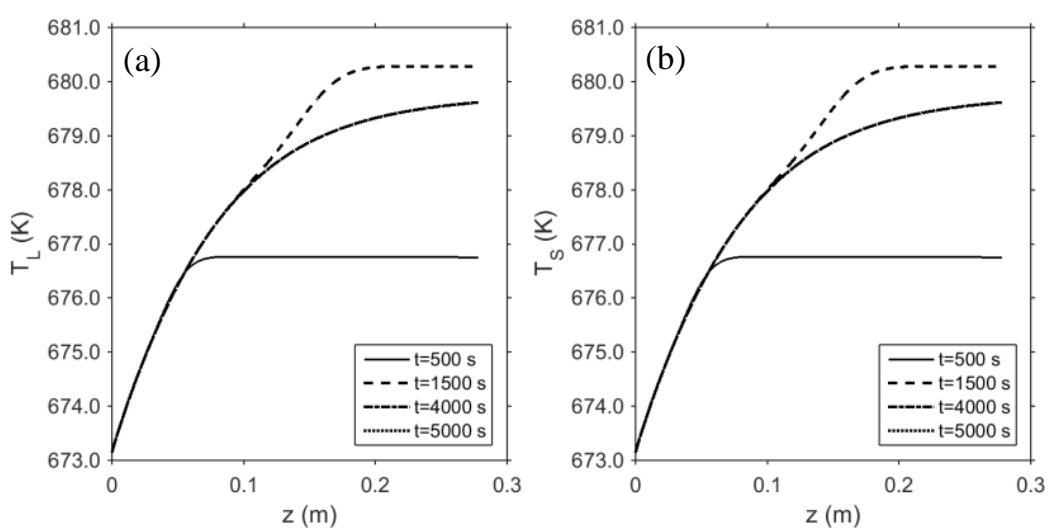

Figura 4: Perfis de temperatura das fases (a) líquida e (b) sólida no reator em regime transiente.

\section{Perfis Concentração e Temperatura em Diferentes Posições no Reator}

Uma observação comum aos perfis demonstrados na Figura 1 a Figura 4 é a sobreposição das curvas no tempo de $4000 \mathrm{~s}$ por aquelas no tempo de $5000 \mathrm{~s}$. Isso ocorre em virtude da concentração não mais variar com o tempo, ou seja, do processo ter atingido o regime estacionário.

$\mathrm{Na}$ Figura 5 os perfis de concentração de hidrogênio e enxofre em diferentes pontos no reator em função do tempo são apresentados. Pode-se verifica a transição do regime de operação transiente para o estacionário à medida que a concentração nos perfis passa a ser constante ao longo do tempo. Na saída do reator, $\mathrm{z}=0,278 \mathrm{~m}$, pode-se notar o momento em que o perfil de concentração atinge o regime estacionário a partir de 4000 segundos.

De maneira similar aos perfis de concentração, analisaram-se os perfis de temperatura da fase líquida com a evolução do tempo em diferentes pontos no reator, que são mostrados na Figura 6. Percebe-se a tendência de elevação da temperatura à medida que se aproxima da saída do reator, além dos diferentes tempos em que o regime estacionário é alcançado nas diferentes posições do reator. 

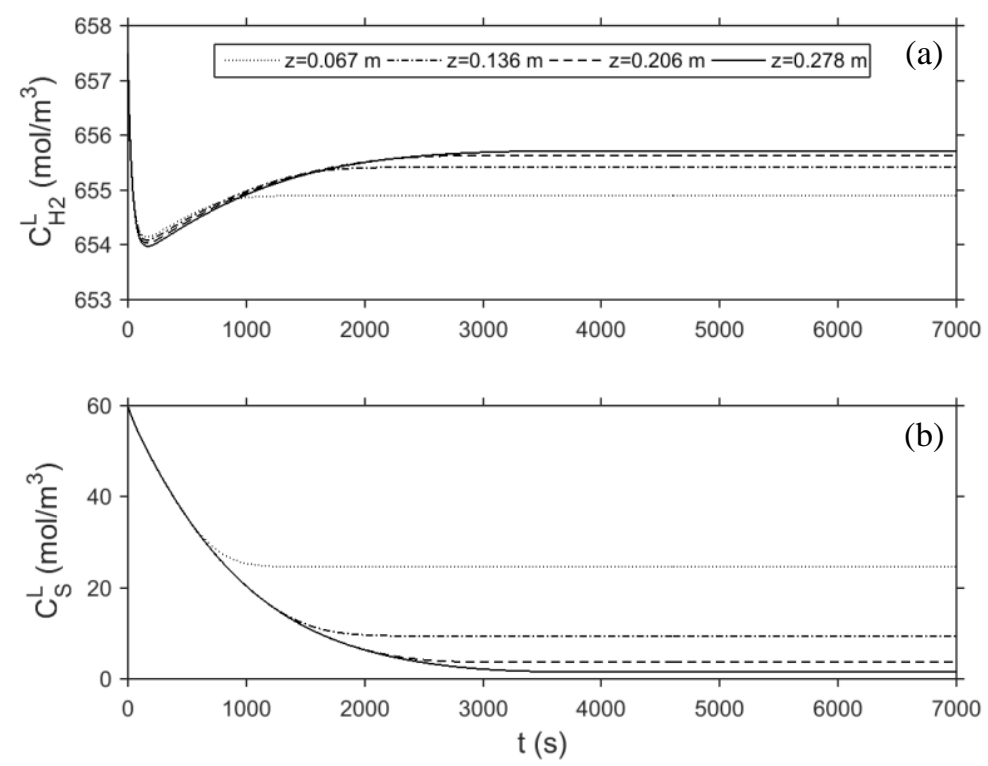

Figura 5: Perfis de concentração de hidrogênio e enxofre na fase líquida em função do tempo e diferentes posições no leito catalítico.

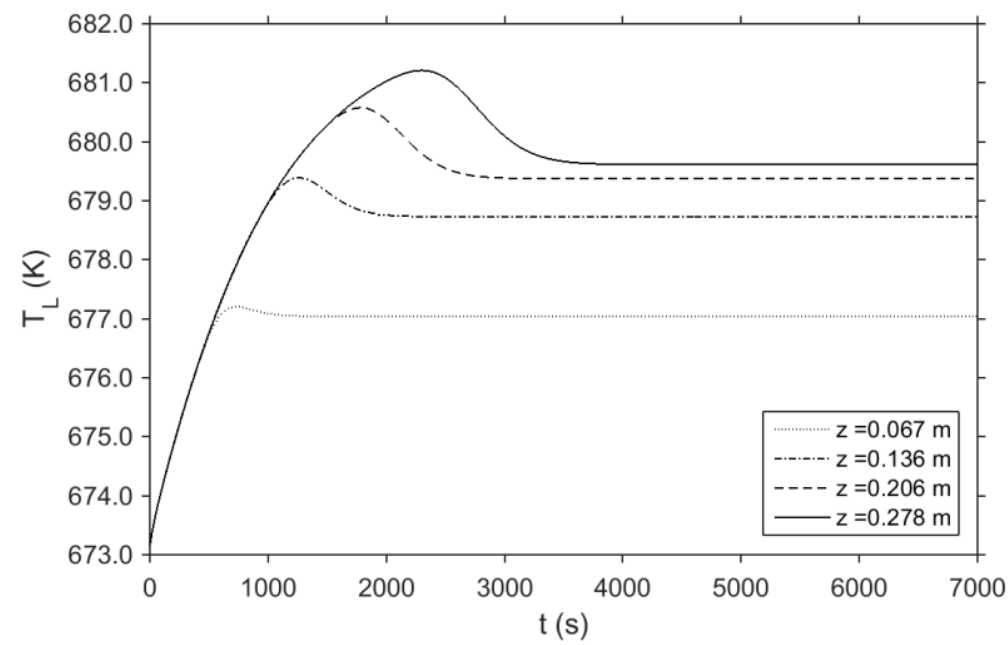

Figura 6: Perfis de concentração de hidrogênio e enxofre na fase líquida em função do tempo e diferentes posições no leito catalítico.

\section{Efeito da Temperatura, Pressão e LHSV no Desempenho do Reator}

Utilizou-se o modelo para simular o desempenho do reator TBR em diferentes condições de operação a fim de estudar as influências dessas variáveis na remoção de enxofre. Os gráficos na Figura 8 foram gerados em 4-10 MPa, LHSV $=1,39 \mathrm{E}-4-4,17 \mathrm{E}-4 \mathrm{~s}^{-1}\left(0,5-1,5 \mathrm{~h}^{-1}\right)$ e T $=335$ $400^{\circ} \mathrm{C}$. 

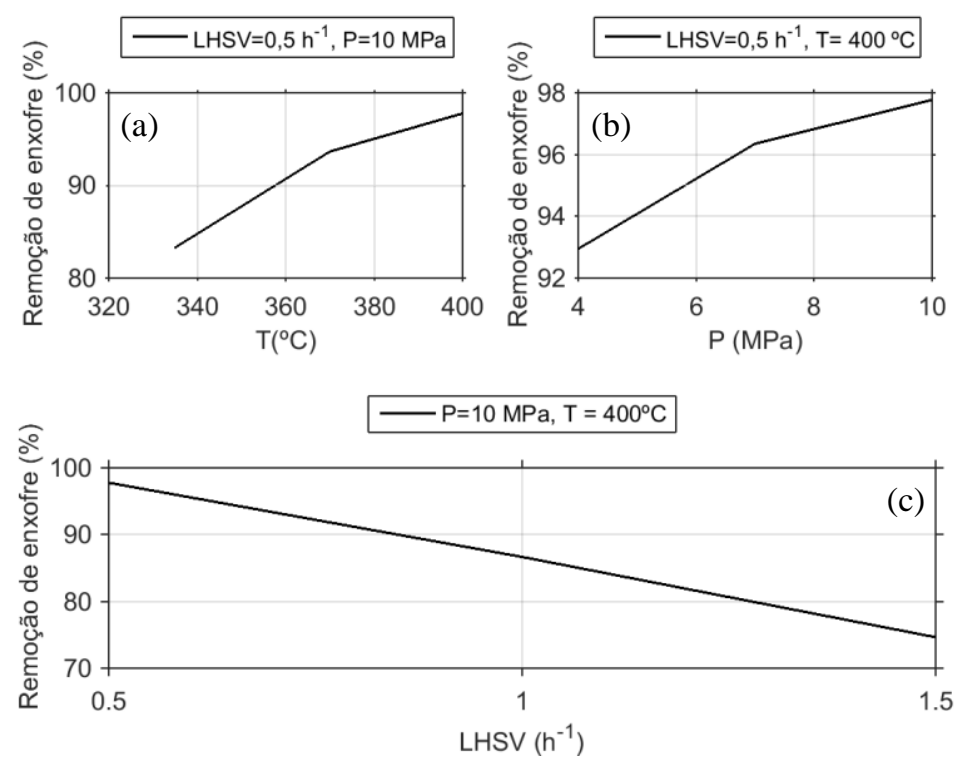

Figura 7: Resultados da simulação de conversão de HDS na saída do reator $(z=0,278 \mathrm{~m})$ e no tempo de $5000 \mathrm{~s}$ em diferentes (a) temperaturas, (b) pressões e (c) velocidades espacial líquida.

Pode-se verificar que a conversão de enxofre aumentou conforme a temperatura e a pressão aumentam, pois essas variáveis tem um efeito positivo sobre a constante cinética, portanto, a taxa de reação também aumenta. Contudo, o aumento da LHSV tem um efeito oposto, pois, conforme a LHSV é aumentada, o tempo de residência diminui, isso significa que a reação tem menos tempo para ocorrer e menos reagente é convertido.

A fim de avaliar o efeito da incerteza dos valores de parâmetros sobre a conversão de HDS, utilizou-se a metodologia abordada por [23] para realizar a análise de sensibilidade do modelo do reator. O cálculo para análise de sensibilidade da conversão de HDS foi realizado levando em consideração à perturbação de $10 \%$ nas variáveis de processo: temperatura $(\mathrm{T})$; pressão total $(\mathrm{P})$; e velocidade espacial horária líquida (LHSV). Os valores-base tomados para essas variáveis foram àqueles referentes à condição de melhor conversão de $\mathrm{HDS}\left(\mathrm{T}=400^{\circ} \mathrm{C}, \mathrm{P}=10 \mathrm{MPa} \mathrm{e}\right.$ LHSV $=1,39 \mathrm{E}-4 \mathrm{~s}^{-1}$, ver Tabela 7).

A Figura 8 mostra o resultado gráfico da análise de sensibilidade da conversão de enxofre. É perceptível que a variável de processo que afeta acentuadamente a conversão do enxofre é a temperatura, seguida da velocidade espacial (LHSV) e, por fim, com menos intensidade a pressão. Além disso, percebe-se que o efeito na sensibilidade da conversão de enxofre é maior no início do reator e se torna menos pronunciado à medida que se percorre o leito catalítico em decorrência da redução da taxa de reação.

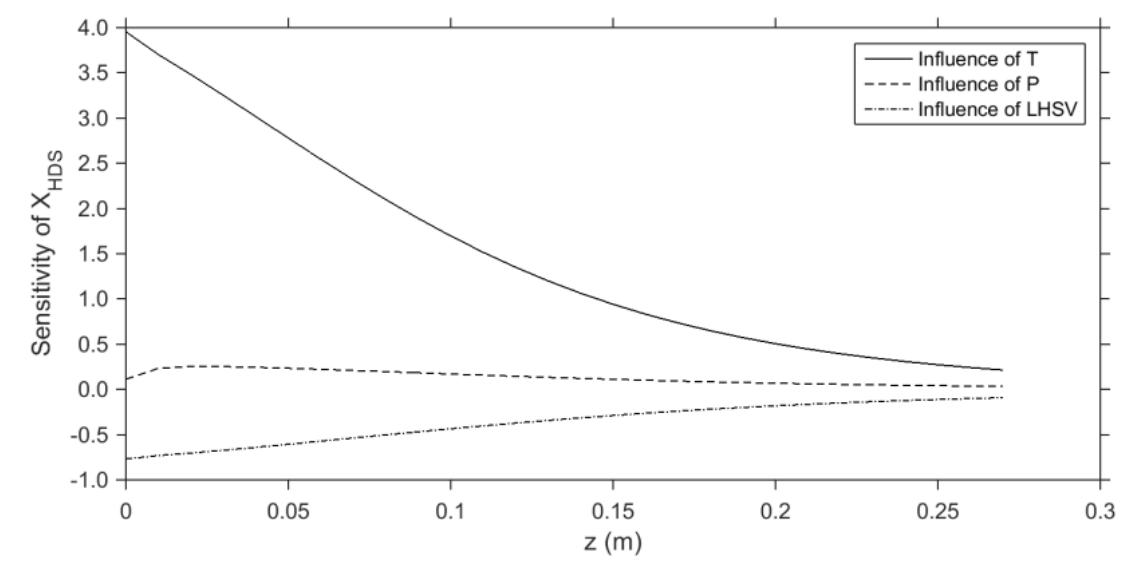

Figura 8: Influência da temperatura $(T)$, pressão total $(P)$ e velocidade espacial horária líquida (LHSV) na conversão de HDS na fase líquida ao longo do comprimento do reator. 


\section{CONCLUSÃO}

No presente trabalho, a modelagem matemática heterogênea de um reator de leito gotejante adiabático e unidimensional foi desenvolvida em regime transiente. Esse modelo descreveu a influência da inibição de $\mathrm{H}_{2} \mathrm{~S}$ na conversão de enxofre, por meio do uso da taxa de reação química do tipo Langmuir-Hinshelwood.

O modelo do reator TBR representou quantitativamente bem os resultados de conversão de HDS experimentais apresentando um erro relativo variando entre $1,15 \%$ e $9,43 \%$. Sendo assim, foram desempenhadas as simulações transientes para gerar os perfis de concentração das espécies envolvidas na reação e de temperatura do reator em função do comprimento do leito.

A avaliação dos efeitos das variáveis de processo no modelo mostrou que a conversão de HDS aumenta com a temperatura e a razão $\mathrm{H}_{2}$ /alimentação, mas diminui com LHSV. Sobretudo, a temperatura mostrou impactar mais significativamente a conversão de enxofre no reator TBR.

\section{NOMENCLATURA}

\begin{tabular}{|c|c|c|}
\hline$a_{L}$ & Área interfacial gás-líquido & $\mathrm{m}^{-1}$ \\
\hline$a_{S}$ & $\begin{array}{l}\text { Área interfacial líquido- } \\
\text { sólido }\end{array}$ & $\mathrm{m}^{-1}$ \\
\hline$C_{P_{L}}$ & $\begin{array}{l}\text { Capacidade calorífica da fase } \\
\text { líquida }\end{array}$ & $\mathrm{J} \mathrm{kg}^{-1} \mathrm{~K}^{-1}$ \\
\hline$C_{P_{S}}$ & $\begin{array}{l}\text { Capacidade calorífica do } \\
\text { catalisador }\end{array}$ & $\mathrm{J} \mathrm{kg}^{-1} \mathrm{~K}^{-1}$ \\
\hline$k_{i}^{S}$ & $\begin{array}{l}\text { Coeficiente de transferência } \\
\text { de massa líquido-sólido da } \\
\text { espécie i }\end{array}$ & $\mathrm{m} \mathrm{s}^{-1}$ \\
\hline$C_{i}^{L}$ & $\begin{array}{l}\text { Concentração da espécie } i \text { na } \\
\text { fase líquida }\end{array}$ & $\mathrm{mol} \mathrm{m}^{-3}$ \\
\hline$C_{i}^{S}$ & $\begin{array}{l}\text { Concentração da espécie } i \text { no } \\
\text { interior do catalisador }\end{array}$ & $\mathrm{mol} \mathrm{m}^{-3}$ \\
\hline$\Delta H_{R, H D S}$ & $\begin{array}{l}\text { Calor global do processo de } \\
\text { HDS }\end{array}$ & $\mathrm{J} \mathrm{mol}^{-1}$ \\
\hline$H_{i}$ & $\begin{array}{l}\text { Coeficiente de Henry da } \\
\text { espécie } i\end{array}$ & $\mathrm{~J} \mathrm{~m}^{-2} \mathrm{~K}^{-1} \mathrm{~s}^{-1}$ \\
\hline$h_{L S}$ & $\begin{array}{l}\text { Coeficiente de transferência } \\
\text { de calor sólido-líquido }\end{array}$ & $\mathrm{J} \mathrm{m}^{-2} \mathrm{~K}^{-1} \mathrm{~s}^{-1}$ \\
\hline$k_{i}^{L}$ & $\begin{array}{l}\text { Coeficiente de transferência } \\
\text { de massa gás-líquido da } \\
\text { espécie i }\end{array}$ & 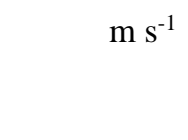 \\
\hline$K_{\mathrm{H}_{2} \mathrm{~S}}$ & $\begin{array}{l}\text { Constante de equilíbrio de } \\
\text { adsorção de } \mathrm{H}_{2} \mathrm{~S}\end{array}$ & $\mathrm{~m}^{3} / \mathrm{mol}$ \\
\hline$k_{H D S}$ & $\begin{array}{l}\text { Constante de taxa de reação } \\
\text { de HDS }\end{array}$ & $\begin{array}{l}\left(\mathrm{mol} \mathrm{m}^{-3}\right)^{1-\mathrm{n}} \\
\left(\mathrm{m}^{3} \mathrm{~kg}^{-1} \mathrm{~s}^{-1}\right) \\
\left(\mathrm{mol} \mathrm{m}^{-3}\right)\end{array}$ \\
\hline$p_{i}^{G}$ & Pressão parcial da espécie i & \\
\hline$R$ & $\begin{array}{l}\text { Constante universal dos } \\
\text { gases }\end{array}$ & $\mathrm{J} \mathrm{mol}^{-1} \mathrm{~K}^{-1}$ \\
\hline$r_{H D S}^{\prime}$ & $\begin{array}{l}\text { Taxa de reação por unidade } \\
\text { de massa de catalisador }\end{array}$ & $\mathrm{mol} \mathrm{kg} \mathrm{kg}^{-1} \mathrm{~s}^{-1}$ \\
\hline$T_{L}$ & Temperatura da fase líquida & $\mathrm{K}$ \\
\hline$T_{S}^{S}$ & $\begin{array}{l}\text { Temperatura na superfície do } \\
\text { catalisador }\end{array}$ & K \\
\hline
\end{tabular}




$\begin{array}{llr}t & \text { Tempo } & \mathrm{s} \\ u_{G} & \text { Velocidade superficial da } & \mathrm{m} \mathrm{s}^{-1} \\ & \text { fase gás } & \\ u_{L} & \begin{array}{l}\text { Velocidade superficial da } \\ \text { fase líquida }\end{array} & \mathrm{m} \mathrm{s}^{-1} \\ & \text { Coordenada axial do reator } & \mathrm{m}\end{array}$

\section{Letras gregas}

\begin{tabular}{|c|c|c|}
\hline${ }_{B}$ & Porosidade do leito catalítico & $m^{3} m^{-3}$ \\
\hline$\varepsilon_{G}$ & $\begin{array}{l}\text { Fração volumétrica de gás por } \\
\text { volume do reator }\end{array}$ & $\mathrm{m}^{3} \mathrm{~m}^{-3}$ \\
\hline$\varepsilon_{L}$ & $\begin{array}{l}\text { Fração volumétrica de líquido por } \\
\text { volume do reator }\end{array}$ & $\mathrm{m}^{3} \mathrm{~m}^{-3}$ \\
\hline$\varepsilon_{P}$ & $\begin{array}{l}\text { Porosidade da partícula do } \\
\text { catalisador }\end{array}$ & 3 \\
\hline$\eta_{H D S}$ & $\begin{array}{l}\text { Fator de efetividade do catalisador } \\
\text { para o processo de HDS }\end{array}$ & \\
\hline$v_{i}$ & $\begin{array}{l}\text { Coeficiente estequiométrico da } \\
\text { espécie } i\end{array}$ & $\mathrm{~m}^{3} \mathrm{~mol}^{-}$ \\
\hline$\rho_{B}$ & Massa especifica do leito catalítico & $\mathrm{kg} \mathrm{m}^{-3}$ \\
\hline$\rho_{L}$ & Massa especifica da fase líquida & $\mathrm{kg} \mathrm{m}^{-3}$ \\
\hline & Massa especifica do catalisador & $\mathrm{kg} \mathrm{m}^{-3}$ \\
\hline
\end{tabular}

\section{Subscritos}

enx Compostos sulfurados

$\mathrm{H}_{2} \quad$ Hidrogênio

$\mathrm{H}_{2} \mathrm{~S} \quad$ Sulfeto de hidrogênio

G Fase gás

$L \quad$ Fase líquida ou interface gás-líquido

$S \quad$ Fase sólida ou interface líquido-sólido

\section{REFERÊNCIAS BIBLIOGRÁFICAS}

1. BP - British Petroleum. BP Statistical Review of World Energy [Internet]. Londres: BP Statistical Review of World Energy; 2015 Jun [Acessado em 2015 jun 11]. Disponível em: http://www.bp.com/.

2. Villasana Y, Escalante Y, Nuñez, JER, Méndez FJ, Ramírez S, Luis-Luis MA, Cañizales E, Ancheyta J, Brito JL. Maya crude oil hydrotreating reaction in a batch reactor using alumina-supported NiMo carbide and nitride as catalysts. Catal Today. 2014 Mar;220-222:318-326, doi: 10.1016/j.cattod.2013.10.025

3. Liu B, Zhao Z, Wang D, Liu J, Chen Y, Li T, Duan A, Jiang G. A theoretical study on the mechanism for thiophene hydrodesulfurization over zeolite L-supported sulfide Co-Mo catalystys: Insight into the hydrodesulfurization over zeolite-based catalysts. Comput Theor Chem. 2015 Jan;1052:47-57, doi: 10.1016/j.comptc.2014.12.001

4. Puello-Polo E, Ayala-G M, Brito JL. Sulfidability and thiophene hydrodesulfurization activity of supported NiMo carbides. Catal Commun. 2014 Aug; 53:9-14, doi: 10.1016/j.catcom.2014.04.018

5. Valencia D, Klimova T, García-Cruz I. Aromaticity of five- and six-membered heterocycles present in crude oil - An electronic description for hydrotratment process. Fuel. 2012 Oct;100:177-185, doi: 10.1016/j.fuel.2012.05.011

6. Martínez J, Ancheyta J., Modeling the kinetics of parallel thermal and catalytic hydrotreating of heavy oil. Fuel. 2014 Dec;138:27-36, doi: 10.1016/j.fuel.2014.02.013 
7. Ferreira AS, Nicoletti MC, Bertini JR, Giordano RC, Methodology for inferring kinetic parameters of diesel oil HDS reaction based on scarce experimental data. Comput Chem Eng. 2013 Jan;48:58-73, doi: 10.1016/j.compchemeng.2012.08.004

8. Heidari A, Hashemabadi SH. CFD study of diesel oil hydrotreating process in the non-isothermal trickle bed reactor. Chem Eng Res Des. 2015 Feb; 94:549-564, doi: ${ }^{\circ} 10.1016 /$ j.cherd.2014.09.016

9. Ranade VV, Chaudhari, RV, Gunjal PR. Trickle Bed Reactor: Engineering and Applications. Amsterdam: Elsevier; 2011. 284 p.

10. Castillo-Araiza CO, Chávez G, Dutta A, Reyes JA. de los, Nuñez S, García-Martínez JC. Role of Pt$\mathrm{Pd} / \gamma$-Al2O3 on the HDS of 4,6-DMBT: kinetic modeling and contribution analysis. Fuel Process Technol. 2015 Apr; 132:164-172, doi: 10.1016/j.fuproc.2014.12.028

11. Kim T, Ali SA, Alhooshani K., Park J, Al-Yami M, Yoon S, Mochilda I. Analysis and deep hydrodesulfurization reactivity of Sudi Arabian gas oils. J Ind Eng Chem. 2013 Sep;19(5):1577-1582, doi: ${ }^{\circ} 10.1016 /$ j.jiec.2013.01.025

12. Rodriguez MA, Elizalde I, Ancheyta J. Comparison of kinetic and reactor models to simulate a tricklebed bech-scale reactor for hydrodesulfurization of VGO. Fuel. 2012 Oct; 100:91-99, doi: 10.1016/j.fuel.2012.01.061

13. Mederos FS, Ancheyta J, Chen J. Review on criteria to ensure ideal behaviors in trickle-bed reactors. Appl Catal A-Gen. 2009 Feb;355 (1-2):1-19, doi: 10.1016/j.apcata.2008.11.018

14. Rodríguez MA, Elizalde I, Ancheyta J. Modeling the performance of a bech-scale reactor sustaining HDS and HDM of heavy crude oil at moderate conditions. Fuel. 2012 Oct; 100: 152-162, doi: 10.1016/j.fuel.2012.03.019

15. Xu M, Liu H, Ji, S, Li C. Intensification of deep hydrodesulfurization through a two-stage combination of monolith and trickle bed reactors. Chinese J Chem Eng. 2014 Aug;22(8), 888-897, doi: 10.1016/j.cjche.2014.06.012

16. Mederos FS., Ancheyta J, Elizalde I. Dynamic modeling and simulation of hydrotreating of gas oil obtained from heavy crude oil. Appl Catal A-Gen. 2012 May;425-426:13-27, doi: 10.1016/j.apcata.2012.02.034

17. Jarullah AT, Mujtaba IM, Wood AS. Kinetic parameter estimation and simulation of trickle-bed reactor for hydrodesulfurization of crude oil. Chem Eng Sci. 2011 Mar;66(5):859-871, doi: 10.1016/j.ces.2010.11.016

18. Müller ALH, Picoloto RS, Mello PA, Ferrão MF, Santos MFP dos, Guimarães RCL, Müller EI., Flores, EMM. Total sulfur determination in residues of crude oil distillation using FT-IR/ATR and variable selection methods. Spectrochim Acta A. 2012 Apr; 89, 82-87, doi: 10.1016/j.saa.2011.12.001

19. Speight JG. The desulfurization of heavy oils and residua. 2nd ed. New York: Marcel Dekker; 2000. $480 \mathrm{p}$.

20. Ancheyta J. Modeling and Simulation of Catalytic Reactor Petroleum Refining. New Jersey: Wiley; 2011, 523 p.

21. Chacón R, Canale A, Bouza A, Sánchez, Y. Modeling of a three-phase reactor for bitumen-derived gas oil hydrotreating. Braz J Chem Eng. 2012 Mar;29(1):135-146, doi: 10.1590/S010466322012000100015

22. Schiesser WE, Griffiths GW. A compendium of partial differential equation models. Cambridge New York: University Press; 2009. 476 p.

23. Perlingeiro CAG. Engenharia de Processos, Análise, Simulação, Otimização e Síntese de Processos Químicos. São Paulo: Edgard Blucher, 2005, 198 p. 